\title{
P310: The blood accidents exposure (aes) in principal hospital dakar (hpd): about 152 cases collected in 12 years
}

\author{
PS Ba ${ }^{1 *}$, B Fall ${ }^{2}$, FK Soumah-Mbaye ${ }^{1}$, KM BA-Fall', PS Mbaye ${ }^{1}$ \\ From 2nd International Conference on Prevention and Infection Control (ICPIC 2013) \\ Geneva, Switzerland. 25-28 June 2013
}

\section{Introduction}

Blood exposure accidents (BEA) are a risk to all personnel of the health services. Today, support for BEA is well codified. In Senegal, a strategy implemented by ISAARV provides rapid and optimized response to these accidents, in order to reduce the risk of HIV seroconversion.

\section{Objectives}

- Specify frequency, circumstances and place of occurrence of BEA among staff, - Highlight the attitudes and practices of personnel during blood exposure situations, - Evaluate the management of these accidents.

\section{Methods}

Retrospective study of all cases reported to the BEA Principal Hospital in Dakar during the period January 2001 - December 2012. Reporting cases of BEA was made in a register in health service staff with collection of a declaration. Declaration forms of all cases were compiled and analyzed in Epi Info version 6.4.

\section{Results}

One hundred and fifty two (152) cases of BSE have been reported and were taken care of. All personal health services were concerned with a predominance of nurses (44.1\%). More than half (59.6\%) were vaccinated against hepatitis B at the time of the accident. Needlestick injuries were the most frequently encountered accidents (86.5\%) followed bz the taking of samples $(44.1 \%)$ or the placement of infusions (22\%). Only $55.9 \%$ of the victims were wearing gloves at the time of the accident. After the accident, $49.2 \%$ of victims washed their hands and used disinfectants recommended in case of BEA. The source patient was identified in $78 \%$ of cases, with 15 cases of HIV positive patients. In the evaluation, $88.1 \%$ were intermediate risk of BEA. Chemoprophylaxis was introduced in $84.7 \%$ of cases, treatment did not exceed 2 days except for 13 of the 15 victims of BEA whose source patients were HIV positive at the time of the accident. No seroconversions were noted.

\section{Conclusion}

This study has made it possible to make recommendations for effective prevention of the BEA, in a broader context of risk management and safety at work.

\section{Disclosure of interest}

None declared.

Author details

${ }^{1}$ Medicine Department, HPD, Dakar, Senegal. ${ }^{2}$ Federation laboratories, HPD, Dakar, Senegal.

Published: 20 June 2013

doi:10.1186/2047-2994-2-S1-P310

Cite this article as: Ba et al:: P310: The blood accidents exposure (aes) in principal hospital dakar (hpd): about 152 cases collected in 12 years. Antimicrobial Resistance and Infection Control 2013 2(Suppl 1):P310.

${ }^{1}$ Medicine Department, HPD, Dakar, Senegal

Full list of author information is available at the end of the article

(c) 2013 Ba et al; licensee BioMed Central Ltd. This is an Open Access article distributed under the terms of the Creative Commons 